\title{
Impact of Modified Transesophageal Echocardiography on Mortality and Stroke after Cardiac Surgery: A Large Cohort Study
}

\author{
Wouter W. Jansen Klomp, ${ }^{1,2}$ Carl G. M. Moons, ${ }^{2}$ \\ Arno P. Nierich, ${ }^{3}$ George J. Brandon Bravo Bruinsma, ${ }^{4}$ Arnoud W. J. van't Hof, ${ }^{1,5}$ \\ Jan G. Grandjean, ${ }^{6}$ and Linda M. Peelen ${ }^{2,7}$ \\ ${ }^{1}$ Department of Cardiology, Isala, Dokter van Heesweg 2, 8025 AB Zwolle, Netherlands \\ ${ }^{2}$ Department of Clinical Epidemiology, Julius Center for Health Sciences and Primary Care, \\ University Medical Center Utrecht, P.O. Box 85500, 3508 GA Utrecht, Netherlands \\ ${ }^{3}$ Department of (Thoracic) Anesthesia and Intensive Care, Isala, Dokter van Heesweg 2, 8025 AB Zwolle, Netherlands \\ ${ }^{4}$ Department of Cardiothoracic Surgery, Isala, Dokter van Heesweg 2, 8025 AB Zwolle, Netherlands \\ ${ }^{5}$ Department of Cardiology, Maastricht University Medical Center, P.O. Box 5800, 6202 AZ Maastricht, Netherlands \\ ${ }^{6}$ MIRA Institute for Biomedical Technology and Technical Medicine, University of Twente, P.O. Box 217, 7500 AE Enschede, Netherlands \\ ${ }^{7}$ Department of Anesthesiology, University Medical Center Utrecht, P.O. Box 85500, 3508 GA Utrecht, Netherlands
}

Correspondence should be addressed to Wouter W. Jansen Klomp; w.w.jansen.klomp@isala.nl

Received 21 April 2017; Revised 27 June 2017; Accepted 18 July 2017; Published 11 September 2017

Academic Editor: Albert G. Hakaim

Copyright (c) 2017 Wouter W. Jansen Klomp et al. This is an open access article distributed under the Creative Commons Attribution License, which permits unrestricted use, distribution, and reproduction in any medium, provided the original work is properly cited.

\begin{abstract}
The aim of this study was to investigate the impact of perioperative screening with modified transesophageal echocardiography (A-View method). We compared, in consecutive patients who underwent cardiac surgery between 2006 and 2014, 30-day mortality and in-hospital stroke incidence, operated either with perioperative modified TEE screening (intervention group) or only with conventional TEE screening (control group). Of the 8,605 study patients, modified TEE was applied in 1,391 patients (16.2\%). Patients in the intervention group were on average older (71 versus 68 years, $p<0.001)$ and more often females $(31.0 \%$ versus $28.0 \%, p<0.001$ ) and had a higher predicted mortality (EuroSCORE I: $5.9 \%$ versus $4.0 \%, p<0.001$ ). The observed 30 -day mortality was $2.2 \%$ and $2.5 \%$ in both groups, respectively, with multivariable and propensity-score adjusted relative risks (RRs) of 0.70 (95\% CI: $0.50-1.00, p=0.05)$ and 0.67 (95\% CI: $0.45-0.98, p=0.04)$. In-hospital stroke was $2.9 \%$ and $2.1 \%$ in both groups, respectively, with adjusted RRs of 1.03 (95\% CI: 0.73-1.45) and 1.01 (95\% CI: 0.71-1.43). In patients undergoing cardiac surgery, use of perioperative screening for aortic atherosclerosis with modified TEE was associated with lower postoperative mortality, but not stroke, as compared to patients operated on without such screening.
\end{abstract}

\section{Introduction}

In patients who undergo cardiac surgery, presence of aortic atherosclerosis is associated with an increased risk of postoperative stroke and mortality [1-4]. Atherogenic emboli released during aortic manipulation are thought to play a pivotal role in the pathophysiology of these complications, as these particles can cause cerebral ischemia. Other symptoms associated with these embolic complications are transient ischemic attack (TIA), convulsions, delirium, and postoperative cognitive decline $[5,6]$. Screening for the presence of aortic atherosclerosis allows for a change in the surgical technique if aortic atherosclerosis is diagnosed, aimed at preventing the formation of such emboli. These changes include a different aortic cannulation site, choosing a different position for the aortic cross-clamp, the use of a dispersion cannula, cardiopulmonary bypass (CPB) through femoral or 
subclavian cannulation, or avoidance of CPB in a beatingheart "no touch aorta" procedure [7].

The distal ascending aorta (DAA) is most prone to the development of atherosclerosis [3]. Guidelines stress the importance of perioperative imaging of this part of the aorta with transesophageal echocardiography (TEE) or epiaortic ultrasound (EAU) [8-10]. Although epiaortic ultrasound is a highly accurate test, its use in practice is limited. On the contrary, TEE is widely used and has the advantage that it can be applied before sternotomy, yet visualization of the upper thoracic aorta is limited by the interposition of the air-filled trachea. Due to this so-called "blind-spot" the sensitivity of TEE for the diagnosis of atherosclerosis of the DAA is only $21 \%$ [11].

Echocardiographic visualization of these structures is possible using a modification to TEE, in which a balloon is introduced in the trachea. After inflation of the balloon with saline, the aortic arch and its branches can be accurately visualized through the trachea [12-14]. Previous studies have shown that modified TEE is safe to apply [12], the sensitivity for detection of atherosclerosis of the DAA was 95\% (95\% confidence interval [CI]: 92\%-99\%) [14], and its diagnostic accuracy was significantly better as compared to conventional TEE imaging [15].

Better diagnostic accuracy for modified TEE as compared to conventional TEE does not necessarily imply that it leads to improved care and consequently outcomes of patients $[16,17]$. We therefore studied the impact of using modified TEE screening in patients undergoing cardiothoracic surgery, as compared to not using such screening, on the actual occurrence of postoperative mortality and stroke.

\section{Methods}

2.1. Study Design and Patients. This observational large cohort study was conducted in the Isala Clinics, Zwolle, The Netherlands. All patients undergoing surgery that required a sternotomy between January 1, 2008, and December 31, 2014, were eligible. Excluded were patients aged below $18(N=1)$, patients who did not give informed consent $(N=163)$, those included in a competing study $(N=189)$, and patients who underwent noncardiac surgery $(N=133)$, thoracic stenting $(N=7)$, or reoperations within 30 days after the index surgery (Figure 1) [14]. Patients provided preoperative written informed consent for use of their data for clinical research, and the study conformed to the principals outlined in the Declaration of Helsinki. The institution's ethical committee waived formal evaluation of the study protocol.

\subsubsection{Interventions}

Index Group. Patients underwent TEE examination before sternotomy to screen for atherosclerosis of the proximal ascending aorta (PAA) and descending aorta. Severity of atherosclerosis was graded following Katz' classification (Table 1) [18]. In general, if TEE imaging showed grade three or higher atherosclerosis, modified TEE was added to visualize the DAA and aortic arch (Figure 2) [18]. This technique was described elaborately in previous publications [12-14].
TABLE 1: Katz' classification of aortic atherosclerosis.

\begin{tabular}{lcc}
\hline $\begin{array}{l}\text { Grade } \\
\text { atherosclerosis }\end{array}$ & Aspect & Clinical impact \\
\hline 1 & $\begin{array}{c}\text { Normal appearing aorta } \\
\text { Extensive intimal thickening }\end{array}$ & Normal \\
2 & Atheroma protruding $<5 \mathrm{~mm}$ & \\
\hline 3 & Atheroma protruding $>5 \mathrm{~mm}$ & Diseased \\
4 & Mobile atheroma & \\
5 & & \\
\hline
\end{tabular}

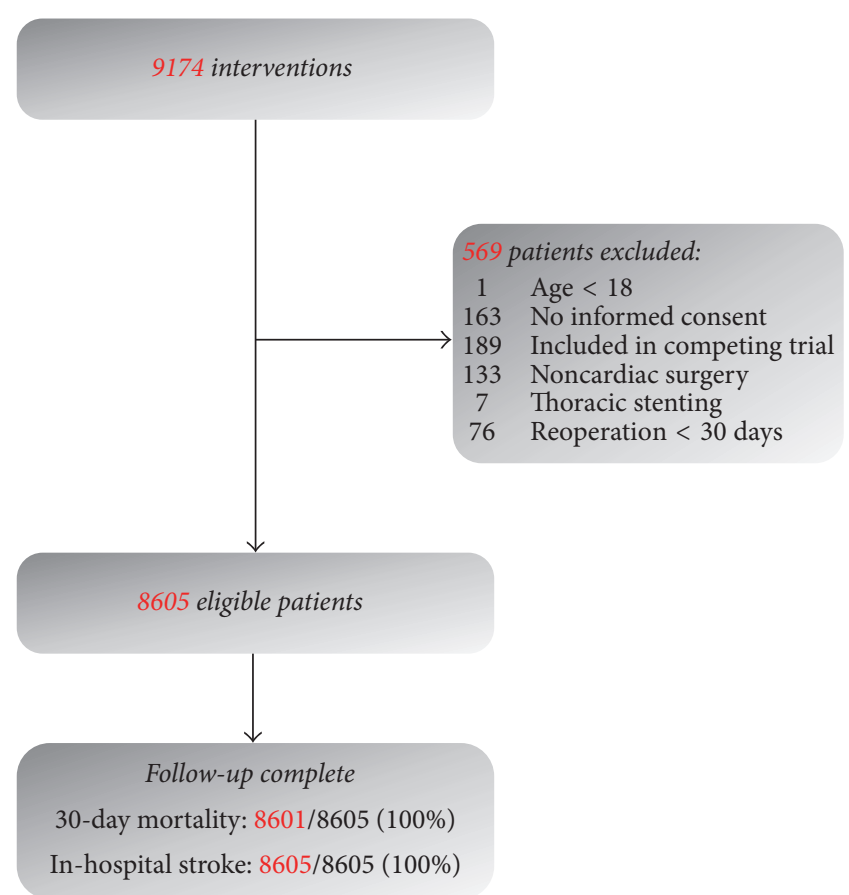

FIGURE 1: Flowchart of patient inclusion.

In short, following a conventional TEE examination the "A-View catheter" (Stroke2prevent, Zwolle, The Netherlands) was introduced via an endotracheal (ET) tube until the $24 \mathrm{~cm}$ marker lined up with a $24 \mathrm{~cm}$ marker on the ET-tube. The correct position of the catheter in the left main bronchus was ascertained, and patients were preoxygenated. Then the balloon was inflated with $20-50 \mathrm{ml}$ of sterile saline, after which imaging could be safely performed within two to three minutes. Three views of the aorta were acquired following a predefined protocol. First, starting from a conventional proximal ascending aortic transesophageal view, the DAA was visualized in a short-axis view by retracting the TEE probe to a depth of $25-30 \mathrm{~cm}$ of the incisors. Then, a long axis view was obtained by rotating the multiplane to $70-120$ degrees. Finally, the aortic arch and its branch vessels were visualized by further retracting the probe in a 0 -degree multiplane angle.

Control Group. The control group included surgical sternotomy patients operated on in the same hospital who did not undergo modified TEE. Perioperative screening with conventional TEE was used standardly in these patients; epiaortic ultrasound was used on indication. 


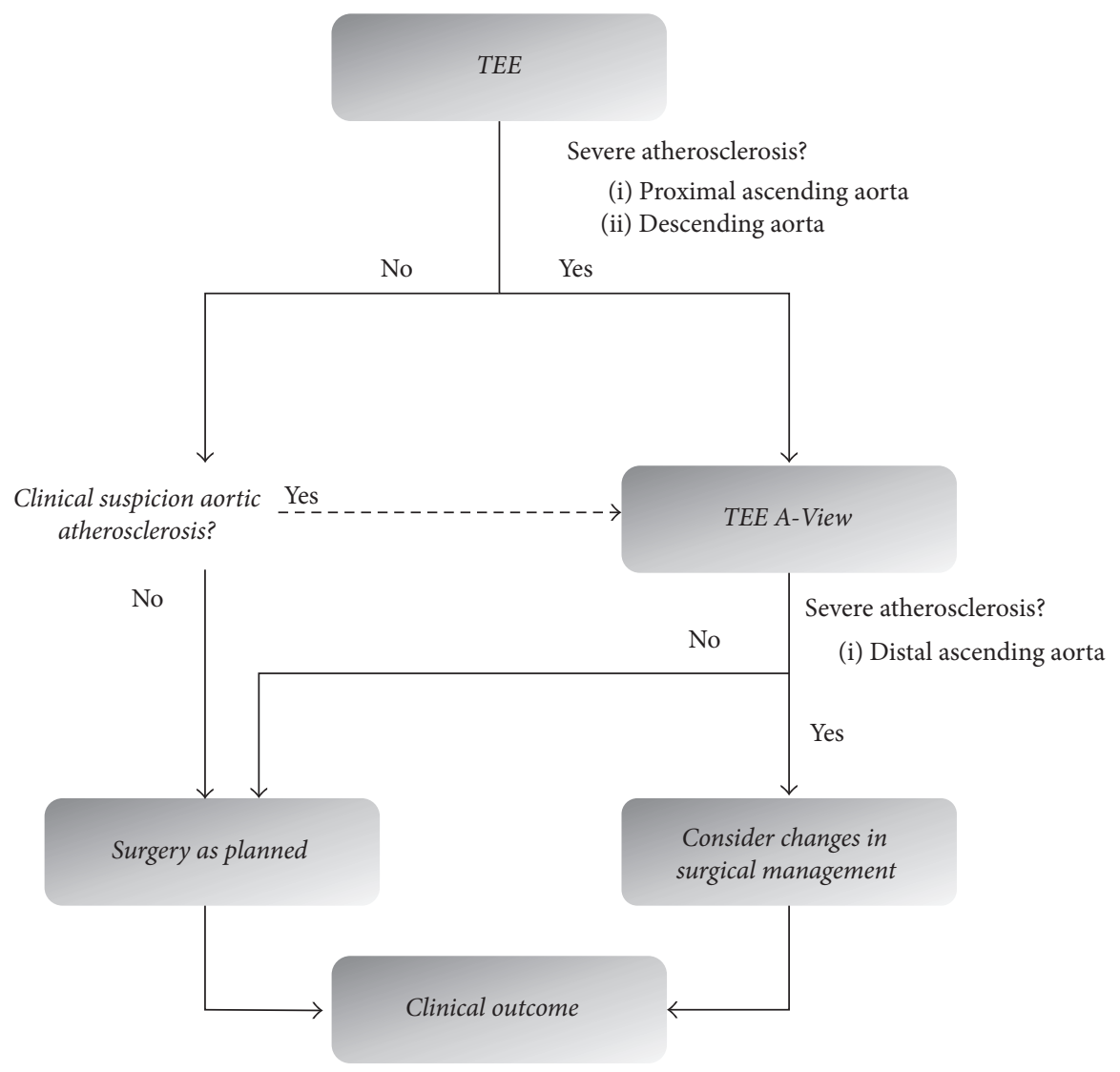

FIGURE 2: Flowchart of decision process to apply preoperative screening with modified TEE.

2.1.2. Endpoints. All-cause 30-day mortality was the endpoint of primary interest. Vital status was obtained through a patients' questionnaire that inquired about adverse events within 30 days after surgery. In patients who did not respond, the general practitioner was contacted. If the vital status was still unknown, the Dutch "Municipal Personal Records Database" was queried.

Secondary endpoint was in-hospital stroke, which was defined as a clinical suspicion of cerebral ischemia lasting longer than 24 hours. The diagnosis was confirmed by an attending neurologist who typically, although not mandatory, assessed presence of cerebral ischemia with cerebral computed tomography (CT) imaging. Stroke was recorded in the ongoing registry; to ascertain that all strokes were identified we also performed a chart review of all patients in whom a cerebral CT was performed during the index-hospitalization.

Finally, as an intermediate endpoint, modifications in the operative management of patients were assessed in both groups. Data was available for two surgical modifications: use of off-pump coronary artery bypass grafting (OPCAB) and cannulation with a different type of cannula ("Select 3D cannula," Medtronic Inc., Minneapolis, MN) [19]. This cannula aims at preventing dislodgement of aortic atheroma by converting the outflow in three streams with a lower velocity [20].
2.1.3. Potential Confounders. Due to our observational cohort design, the allocation to the use of modified TEE was clearly not random. Particularly, patients with a higher risk of having aortic atherosclerosis, based on the TEE results as well as on patient characteristics, were likely more indicated to undergo modified TEE screening during surgery. Since the same characteristics that indicate the use of modified TEE are also associated with a higher risk of postoperative stroke and mortality, the crude estimated effect association of the use of modified TEE (as compared to the control group) and the outcomes was likely distorted by these factors. We therefore considered the following confounders to be adjusted for in the analysis: logistic EuroSCORE, sex, age, hypertension, diabetes mellitus, unstable angina pectoris, previous myocardial infarction, previous percutaneous transluminal coronary angioplasty, previous cardiac surgery, preoperative creatinin, left ventricular ejection fraction (LVEF; $<30 \%$, $30-50 \%$, and $>50 \%$ ), type of surgery (isolated coronary artery bypass grafting [CABG], isolated valve, $\mathrm{CABG}+$ valve, and others), and emergency indication. Age and EuroSCORE were modelled using a log transformation.

\subsection{Statistical Analysis}

2.2.1. Unadjusted Analysis. First, the crude association of the use of modified TEE (intervention) as compared to not using 
TABLE 2: Baseline characteristics comparing patients with and without perioperative screening with modified TEE. BMI $=$ body mass index, $\mathrm{DM}=$ diabetes mellitus, $\mathrm{COPD}=$ chronic obstructive pulmonary disease, $\mathrm{TIA}=$ transient ischemic attack, $\mathrm{PTCA}=$ percutaneous transluminal coronary angioplasty, and LVEF = left ventricular ejection fraction.

\begin{tabular}{|c|c|c|c|}
\hline & $\begin{array}{l}\text { Modified TEE } \\
\quad(N=1,391)\end{array}$ & $\begin{array}{c}\text { Nonmodified TEE } \\
\quad(N=7,214)\end{array}$ & $p$ \\
\hline Age & $71(65-78)$ & $68(61-75)$ & $<0.001$ \\
\hline Female sex & $431(31.0)$ & $2,023(28.0)$ & 0.026 \\
\hline BMI & $27.0(24.9-30.0)$ & $27.0(25.0-30.0)$ & 0.38 \\
\hline \multicolumn{4}{|l|}{ History of } \\
\hline Hypertension & $867(62.3)$ & $3,490(48.4)$ & $<0.001$ \\
\hline $\mathrm{DM}$ & $365(26.2)$ & $1,629(22.6)$ & 0.003 \\
\hline Peripheral atherosclerosis & $264(19.0)$ & $651(9.0)$ & $<0.001$ \\
\hline TIA or stroke & $192(13.8)$ & $545(7.6)$ & $<0.001$ \\
\hline COPD & $255(18.4)$ & $1015(14.1)$ & $<0.001$ \\
\hline Myocardial Infarction & $411(29.5)$ & $2,028(28.1)$ & 0.27 \\
\hline PTCA & $242(17.4)$ & $1,064(14.7)$ & 0.012 \\
\hline Cardiothoracic surgery & $109(7.8)$ & $405(5.6)$ & 0.001 \\
\hline Instable angina & $80(5.8)$ & $350(4.9)$ & 0.16 \\
\hline \multicolumn{4}{|l|}{ LVEF } \\
\hline$>50 \%$ & $870(62.5)$ & $4,725(61.2)$ & \multirow{3}{*}{0.069} \\
\hline $30-50 \%$ & $406(29.2)$ & $1,976(31.3)$ & \\
\hline$<30 \%$ & $115(8.2)$ & $508(7.0)$ & \\
\hline Preoperative creatinin & $87(75-103)$ & $85(73-100)$ & $<0.001$ \\
\hline Logistic EuroSCORE & $5.9(2.9-12.5)$ & $4.0(2.1-8.2)$ & $<0.001$ \\
\hline
\end{tabular}

this technique (control) was assessed for both outcomes using the relative risk (RR) and 95\% CI.

2.2.2. Multivariable Regression. Second, the association regarding the use of modified TEE as compared to the control group was corrected for the above-mentioned confounders, again for both endpoints. Rather than multivariable logistic regression, we used a binomial distribution with a loglink to obtain RRs with a 95\% CI, which are more easily interpretable than odds ratios and are synchronous with the crude association measure $[21,22]$.

2.2.3. Propensity-Score Analysis. Additionally, we corrected for confounding with propensity-score (PS) analysis [23]. Using logistic regression analysis a PS was created, with modified TEE as the dependent variable and the confounders as covariates. After checking that the range of the PS was similar for the exposed and the unexposed and that the score was well balanced over quintiles of the PS, the individual propensity scores were added to a model with only modified TEE status as a covariate.

Analyses were performed in SPSS 22.0 and R version 3.1.0. A $p$ value $<0.05$ was regarded statistically significant throughout this article.

\section{Results}

Of the 8,605 study patients, modified TEE was applied in 1,391 patients (16.2\%). Table 2 summarizes the baseline characteristics in comparison of both groups. The modified TEE (index) group included more females, and patients were on average older. These patients had more comorbid diseases, including hypertension, diabetes mellitus, extracardiac atherosclerosis, previous TIA or stroke, and COPD. Surgery in the index group more often included replacement of the aortic valve, ascending aorta, or (partial) aortic arch (Table 3). Duration of the extracorporeal circulation was similar in both groups, as was the postoperative ventilation time.

3.1. Change in Surgical Management. In the subgroup of patients who underwent coronary revascularization, the use of off-pump techniques was lower in the index group as compared to the control group (9.9\% versus $15.7 \%$; $p<$ 0.001). Aortic cannulation with the "Select 3D cannula" was used more frequently in the modified TEE group than in the control group $(2.5 \%$ versus $0.4 \% ; p<0.001)$.

3.2. Mortality. The EuroSCORE-predicted mortality was $5.9 \%$ in the intervention group and $4.0 \%$ in the control group $(p<0.001)$. The observed 30 -day mortality was $2.2 \%$ and $2.5 \%$ in both groups, respectively (RR: 0.89 ; 95\% CI: $0.61-1.30$, $p=0.55$; Table 4; Figure 3 ). Perioperative screening for aortic atherosclerosis was associated with a lower mortality after both multivariate adjustment (RR 0.70, 95\% CI: 0.48-1.00, $p$ $=0.050)$ and propensity-score adjustment (RR 0.67, 95\% CI: $0.45-0.98, p=0.040)$.

3.3. Stroke. The predicted incidence of stroke was $4.1 \%$ $(1.2 \%-4.8 \%)$ in patients with modified TEE and $2.8 \%$ 
TABLE 3: Operative characteristics comparing patients with and without perioperative screening with modified TEE. * Percentage of patients with isolated coronary revascularization. $\mathrm{CABG}=$ coronary artery bypass grafting, $\mathrm{CK}=$ Creatinin Kinase, and $\mathrm{CKMb}=\mathrm{Creatinin}$ Kinase MB isoenzyme.

\begin{tabular}{|c|c|c|c|}
\hline & $\begin{array}{l}\text { Modified TEE } \\
(N=1,391)\end{array}$ & $\begin{array}{l}\text { Nonmodified TEE } \\
\quad(N=7,214)\end{array}$ & $p$ \\
\hline Emergency indication & $25(1.8)$ & $205(2.8)$ & $<0.001$ \\
\hline \multicolumn{4}{|l|}{ Surgery } \\
\hline CABG & $942(67.7)$ & $5,033(69.8)$ & 0.13 \\
\hline Off-pump CABG & $63(9.9)^{*}$ & $488(15.7)^{*}$ & $<0.001$ \\
\hline Aortic valve surgery & $481(34.6)$ & $1,860(25.8)$ & $<0.001$ \\
\hline Mitral valve surgery & $202(14.5)$ & $1,090(15.1)$ & 0.57 \\
\hline Aortic root replacement & $36(2.6)$ & $164(2.3)$ & 0.48 \\
\hline Ascending aorta replacement & $81(5.8)$ & $246(3.4)$ & $<0.001$ \\
\hline (Partial) arch replacement & $27(1.9)$ & $73(1.0)$ & 0.003 \\
\hline Extracorporeal circulation (min) & $109(75-164)$ & $105(78-153)$ & 0.23 \\
\hline Blood loss during surgery (ml) & $200(150-300)$ & $200(200-350)$ & $<0.001$ \\
\hline \multicolumn{4}{|l|}{ Postoperative } \\
\hline Ventilation & $6(4-9)$ & $5(4-8)$ & 0.54 \\
\hline $\mathrm{CK}(\max )$ & $343(206-692)$ & $332(210-598)$ & 0.61 \\
\hline CKMb (max) & $42(28-68)$ & $35(25-63)$ & 0.003 \\
\hline
\end{tabular}

TABLE 4: Primary outcome measures comparing patients with and without perioperative screening with modified TEE.

\begin{tabular}{lccc}
\hline & Modified TEE & Nonmodified TEE & $(N=7,214)$ \\
& $(N=1,391)$ & $177(2.5)$ & 0.55 \\
Mortality, 30-day & $31(2.2)$ & $155(2.1)$ & 0.067 \\
Stroke, in-hospital & $41(2.9)$ & & \\
\hline
\end{tabular}

$(0.9 \%-3.4 \%)$ in the control group. The observed in-hospital stroke rate was $2.9 \%$ and $2.1 \%$, respectively (RR $1.37 ; 95 \% \mathrm{CI}$ : $0.98-1.93$, $p=0.067$; Table 4; Figure 3). The multivariableand propensity-score adjusted RRs for in-hospital stroke were $1.03(0.73-1.45)$ and $1.01(0.71-1.43)$, indicating that stroke risk is similar in patients with and without modified TEE screening.

\section{Discussion}

This large nonrandomized intervention study showed that perioperative screening for aortic atherosclerosis with modified TEE was associated with a lower mortality after correction for confounding, as compared to patients without screening. The use of modified TEE was also associated with limited changes in the surgical management, but not with a lower incidence of stroke.

The evaluation of any new test should move beyond diagnostic accuracy, since an accurate test does not result in improved patient outcomes per se. First, a test should lead to an improved (or more timely) diagnosis; second it should lead to effective changes in patient management $[16,17,24-$ 27]. Considering modified TEE, we already showed that the diagnosis of aortic atherosclerosis was accurate and that the diagnosis was improved beyond conventional TEE imaging $[14,15]$. Therefore, it was a logical next step to study the clinical effects of this test [7]. An ideal design would be a randomized diagnostic intervention study. However, randomized studies are expensive and time-consuming. Thus, we (first) performed a nonrandomized study to study the association between the use of modified TEE and clinical outcomes.

Modified TEE was used on indication, primarily if atherosclerosis of the proximal ascending aorta (PAA) or descending aorta was observed with conventional TEE. The baseline characteristics clearly reflect this "confounding by indication," as patients with modified TEE screening were on average older and had more comorbid conditions than patients without modified TEE. Although the EuroSCOREpredicted mortality was higher in the former group $(5.9 \%$ versus $4.1 \%, p<0.001$ ), the observed 30 -day mortality was nonsignificantly lower $(2.2 \%$ versus $2.5 \%, p=0.55)$ and its use was associated with a lower propensity-score adjusted 30 day mortality (RR: 0.67; 95\% CI: $0.45-0.98$ ) as compared to the control group. Emboli related death is a common cause for postoperative mortality and may have contributed to this difference [28]. Another explanation for this difference may be that attending anesthesiologists differed not only in the use of modified TEE but also in other aspects of patient management. Since the effect of any diagnostic modality can only be operationalized through the actions of the observer, it would however be incorrect to adjust for the observers. 

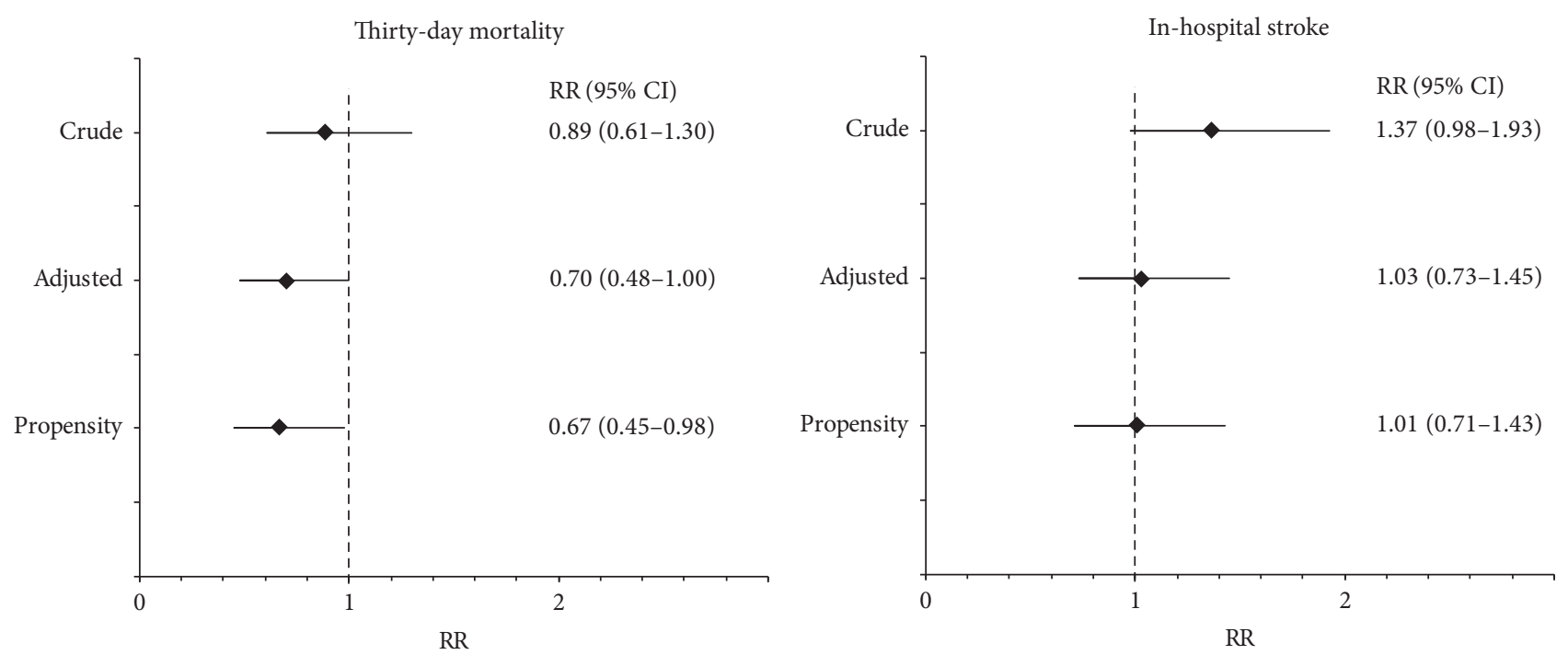

FIGURE 3: Crude, multivariable-adjusted and propensity-score adjusted relative risk of 30-day mortality and in-hospital stroke in patients with preoperative modified TEE screening compared to those without modified TEE.

Both the expected and the observed incidence of stroke were higher in the modified TEE group; after correction for confounding, the stroke incidence was similar in both groups. This contrasted our expectation that modified TEE would be associated with a reduced incidence of stroke. Several explanations can be hypothesized for this finding. First, although we consider the large cohort a strength of this study, potentially we were still underpowered to show statistical significance of a difference in stroke incidence. Second, it is possible that there is still residual confounding because of unmeasured covariates. To conclude that use of modified TEE indeed reduces the risk of stroke, with similar incidences and a power of 0.80 , would require an RCT with inclusion of 3825 patients per group.

Changes in patient outcome can only be achieved through improved patient management. We showed that the use of a so-called "3D dispersion cannula" was higher in the modified TEE group. This catheter is aimed at reducing the sandblasting effects of CPB on the posterior aortic wall by diverting the flow into multiple jets [19]. We also studied the use of off-pump revascularization, which is associated with a reduction in stroke especially in patients with aortic atherosclerosis [29]. Yet, in contrast to our expectations, an off-pump approach was used less often in the modified TEE group. Possibly, screening for aortic atherosclerosis was considered less needed in procedures without aortic manipulations.

It would have been of interest to have a more complete picture of other steps between test application and outcome such as subtle changes in the positioning of the aortic crossclamp or $\mathrm{CPB}$ cannula. These were not collected in the registry and cannot be assessed retrospectively. Also, at the time of this study, we did not yet describe the indications for modified TEE in a protocol and the changes in patient management in the presence of aortic atherosclerosis were not standardized. Meanwhile, we have developed protocol providing a systematic approach for the diagnosis of atherosclerosis and the subsequent considerations in the intraoperative management [30].

Despite these limitations, the results of this hypothesisgenerating study warrant further research to establish the association between modified TEE and patient outcomes [31]. A randomized diagnostic design should be considered to overcome the current limitations associated with "confounding by indication." In such a study, the changes in the surgical management in the presence of aortic atherosclerosis should be further protocolized and registered in detail.
Abbreviations
CABG: Coronary artery bypass grafting
CPB: Cardiopulmonary bypass
CT: $\quad$ Computed tomography
DAA: Distal ascending aorta
ET: $\quad$ Endotracheal
LVEF: Left ventricular ejection fraction
TEE: Transesophageal echocardiography
TIA: Transient ischemic attack
OPCAB: Off-pump cardiopulmonary bypass
PAA: Proximal ascending aorta.

\section{Disclosure}

An earlier version of this work was presented as a poster at the Annual Thoracic Anesthesia Symposium 2016.

\section{Conflicts of Interest}

Dr. Nierich is the inventor of the A-View technique and Chief Medical Officer of Stroke2prevent B.V. (https://www .stroke2prevent.com), during the conduct of the study. All other authors declare that there are no conflicts of interest regarding the publication of this paper. 


\section{References}

[1] V. G. Dávila-Román, S. F. Murphy, N. J. Nickerson, N. T. Kouchoukos, K. B. Schechtman, and B. Barzilai, "Atherosclerosis of the ascending aorta is an independent predictor of longterm neurologic events and mortality," Journal of the American College of Cardiology, vol. 33, no. 5, pp. 1308-1316, 1999.

[2] G. Djaiani, L. Fedorko, M. Borger et al., "Mild to moderate atheromatous disease of the thoracic aorta and new ischemic brain lesions after conventional coronary artery bypass graft surgery," Stroke, vol. 35, no. 9, pp. e356-358, 2004.

[3] J. D. van der Linden, L. Hadjinikolaou, P. Bergman, and D. Lindblom, "Postoperative stroke in cardiac surgery is related to the location and extent of atherosclerotic disease in the ascending aorta," Journal of the American College of Cardiology, vol. 38, no. 1, pp. 131-135, 2001.

[4] J. van der Linden, P. Bergman, and L. Hadjinikolaou, "The topography of aortic atherosclerosis enhances its precision as a predictor of stroke," Annals of Thoracic Surgery, vol. 83, no. 6, pp. 2087-2092, 2007.

[5] D. van Dijk, A. M. A. Keizer, J. C. Diephuis, C. Durand, L. J. Vos, and R. Hijman, "Neurocognitive dysfunction after coronary artery bypass surgery: a systematic review," Journal of Thoracic and Cardiovascular Surgery, vol. 120, no. 4, pp. 632-639, 2000.

[6] J. Bucerius, J. F. Gummert, M. A. Borger et al., "Predictors of delirium after cardiac surgery delirium: effect of beating-heart (off-pump) surgery," Journal of Thoracic and Cardiovascular Surgery, vol. 127, no. 1, pp. 57-64, 2004.

[7] R. M. Engelman and D. T. Engelman, "Strategies and Devices to Minimize Stroke in Adult Cardiac Surgery," Seminars in Thoracic and Cardiovascular Surgery, vol. 27, no. 1, pp. 24-29, 2015.

[8] M. D. Cheitlin, W. F. Armstrong, G. P. Aurigemma et al., "ACC/AHA/ASE 2003 guideline update for the clinical application of echocardiography: summary article: a report of the American College of Cardiology/American Heart Association Task Force on Practice Guidelines (ACC/AHA/ASE Committee to Update the 1997 Guidelines for the Clinical Application of Echocardiography)," Circulation, vol. 108, pp. 1146-1162, 2003.

[9] "Practice guidelines for perioperative transesophageal echocardiography," vol. 112, pp. 1084-1096, 1996.

[10] F. A. Flachskampf, L. Badano, W. G. Daniel et al., "Erratum: Recommendations for transoesophageal echocardiography: Update 2010 (European Journal of Echocardiography," European Journal of Echocardiography, vol. 11, no. 3, pp. 557-576, 2010.

[11] B. Van Zaane, N. P. A. Zuithoff, J. B. Reitsma, L. Bax, A. P. Nierich, and K. G. M. Moons, "Meta-analysis of the diagnostic accuracy of transesophageal echocardiography for assessment of atherosclerosis in the ascending aorta in patients undergoing cardiac surgery," Acta Anaesthesiologica Scandinavica, vol. 52, no. 9, pp. 1179-1187, 2008.

[12] B. van Zaane, A. P. Nierich, W. F. Buhre, G. J. Brandon Bravo Bruinsma, and K. G. M. Moons, "Resolving the blind spot of transoesophageal echocardiography: a new diagnostic device for visualizing the ascending aorta in cardiac surgery," British Journal of Anaesthesia, vol. 98, no. 4, pp. 434-441, 2007.

[13] A. P. Nierich, B. van Zaane, W. F. Buhre, J. Coddens, A. J. Spanjersberg, and K. G. M. Moons, "Visualization of the distal ascending aorta with A-mode transesophageal echocardiography," Journal of Cardiothoracic and Vascular Anesthesia, vol. 22, no. 5, pp. 766-773, 2008.
[14] B. Van Zaane, A. P. Nierich, G. J. B. B. Bruinsma et al., "Diagnostic accuracy of modified transoesophageal echocardiography for pre-incision assessment of aortic atherosclerosis in cardiac surgery patients," British Journal of Anaesthesia, vol. 105, no. 2, pp. 131-138, 2010.

[15] W. W. Jansen Klomp, L. M. Peelen, S. J. Spanjersberg et al., "Added value of modified transoesophageal echocardiography in the diagnosis of atherosclerosis of the distal ascending aorta in cardiac surgery patients," European Heart Journal Cardiovascular Imaging, vol. 15, no. 6, pp. 623-630, 2014.

[16] K. G. Moons, "Criteria for scientific evaluation of novel markers: a perspective," Clinical Chemistry, vol. 56, no. 4, pp. 537-541, 2010.

[17] P. M. Bossuyt, J. B. Reitsma, K. Linnet, and K. G. Moons, "Beyond diagnostic accuracy: the clinical utility of diagnostic tests," Clinical Chemistry, vol. 58, no. 12, pp. 1636-1643, 2012.

[18] E. S. Katz, P. A. Tunick, H. Rusinek, G. Ribakove, F. C. Spencer, and I. Kronzon, "Protruding aortic atheromas predict stroke in elderly patients undergoing cardiopulmonary bypass: experience with intraoperative transesophageal echocardiography," Journal of the American College of Cardiology, vol. 20-21, no. 1, pp. 70-77, 1992.

[19] A. L. Lata, J. W. Hammon, D. D. Deal, D. A. Stump, E. H. Kincaid, and N. D. Kon, "Cannula design reduces particulate and gaseous emboli during cardiopulmonary bypass for coronary revascularization," Perfusion, vol. 26, no. 3, pp. 239-244, 2011.

[20] M. Minakawa, I. Fukuda, J. Yamazaki, K. Fukui, H. Yanaoka, and T. Inamura, "Effect of cannula shape on aortic wall and flow turbulence: Hydrodynamic study during extracorporeal circulation in mock thoracic aorta," Artificial Organs, vol. 31, no. 12, pp. 880-886, 2007.

[21] L. McNutt, C. Wu, X. Xue, and J. P. Hafner, "Estimating the relative risk in cohort studies and clinical trials of common outcomes," The American Journal of Epidemiology, vol. 157, no. 10, pp. 940-943, 2003.

[22] R. H. H. Groenwold, K. G. M. Moons, L. M. Peelen, M. J. Knol, and A. W. Hoes, "Reporting of treatment effects from randomized trials: A plea for multivariable risk ratios," Contemporary Clinical Trials, vol. 32, no. 3, pp. 399-402, 2011.

[23] T. Stürmer, M. Joshi, R. J. Glynn, J. Avorn, K. J. Rothman, and S. Schneeweiss, "A review of the application of propensity score methods yielded increasing use, advantages in specific settings, but not substantially different estimates compared with conventional multivariable methods," Journal of Clinical Epidemiology, vol. 59, no. 5, pp. 437-447, 2006.

[24] C. J. Biesheuvel, D. E. Grobbee, and K. G. M. Moons, "Distraction from randomization in diagnostic research," Annals of Epidemiology, vol. 16, no. 7, pp. 540-544, 2006.

[25] L. Ferrante Di Ruffano, C. J. Hyde, K. J. McCaffery, P. M. M. Bossuyt, and J. J. Deeks, "Assessing the value of diagnostic tests: A framework for designing and evaluating trials," BMJ (Online), vol. 344, no. 7847, article no. e686, 2012.

[26] L. Ferrante Di Ruffano, C. Davenport, A. Eisinga, C. Hyde, and J. J. Deeks, "A capture-recapture analysis demonstrated that randomized controlled trials evaluating the impact of diagnostic tests on patient outcomes are rare," Journal of Clinical Epidemiology, vol. 65, no. 3, pp. 282-287, 2012.

[27] B. M. Reilly and A. T. Evans, "Translating clinical research into clinical practice: impact of using prediction rules to make decisions," Annals of Internal Medicine, vol. 144, no. 3, pp. 201209, 2006. 
[28] P. Emmrich, J. Hahn, V. Ogunlade, K. Geiger, R. Schober, and F. W. Mohr, "Neuropathological findings after cardiac surgery-retrospective study over 6 years," Zeitschrift für Kardiologie, vol. 92, no. 11, pp. 925-937, 2003.

[29] J. Afilalo, M. Rasti, S. M. Ohayon, A. Shimony, and M. J. Eisenberg, "Off-pump vs. on-pump coronary artery bypass surgery: An updated meta-analysis and meta-regression of randomized trials," European Heart Journal, vol. 33, no. 10, pp. 1257-1267, 2012.

[30] W. W. Jansen Klomp, G. J. Brandon Bravo Bruinsma, A. W. J. Hof van 't, J. G. Grandjean, and A. P. Nierich, "A protocol for diagnosis and management of aortic atherosclerosis in cardiac surgery patients," International Journal of Vascular Medicine, vol. 2017, Article ID 1874395, 8 pages, 2017.

[31] W. Jansen Klomp, “(2016, December 2). Thesis. Diagnostic challenges in aortic disease. Gildeprint, Enschede, The Netherlands”. 


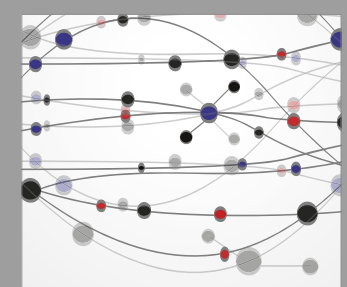

The Scientific World Journal
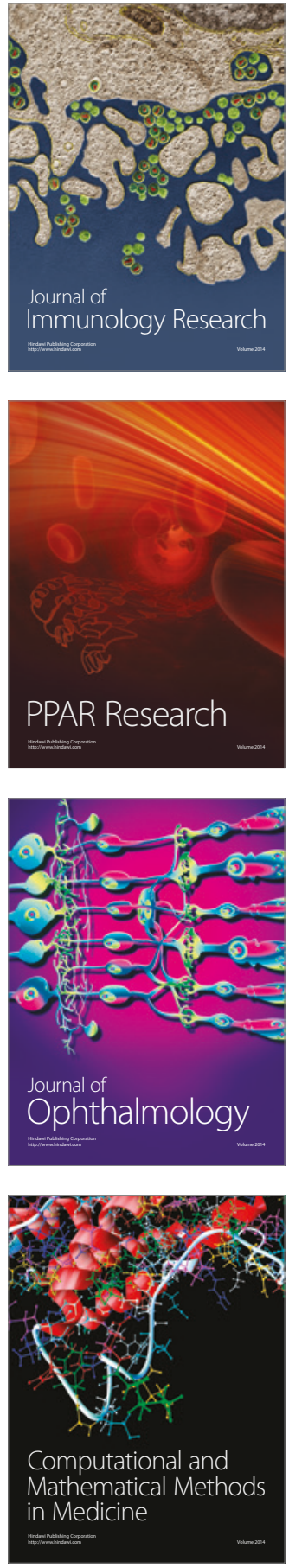

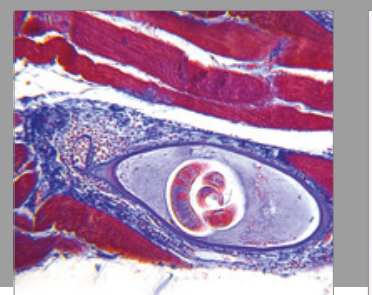

Gastroenterology Research and Practice
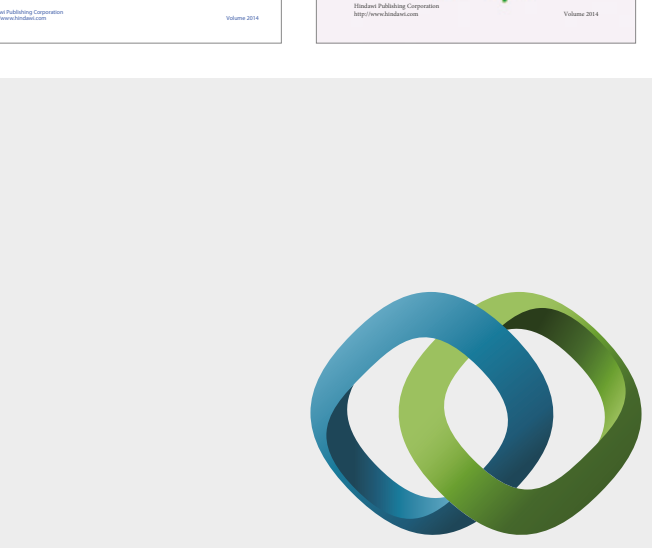

\section{Hindawi}

Submit your manuscripts at

https://www.hindawi.com
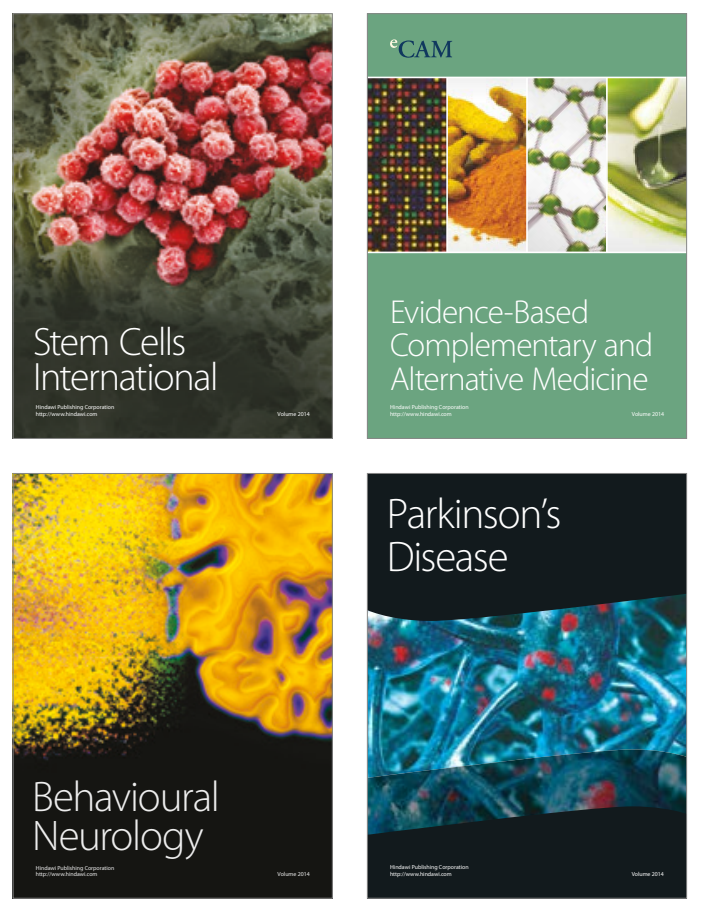
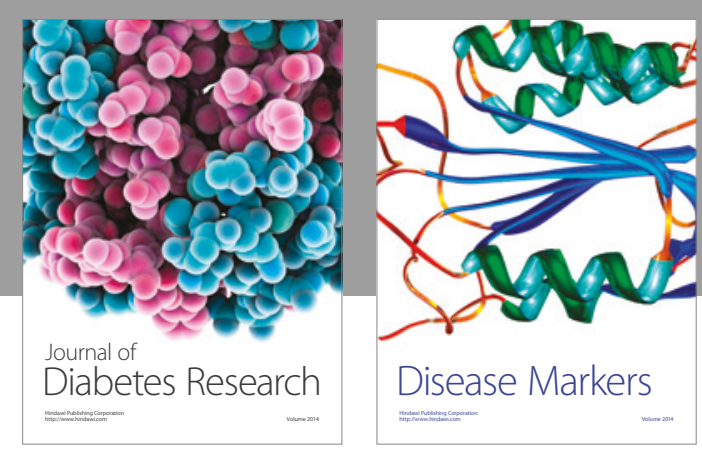

Disease Markers
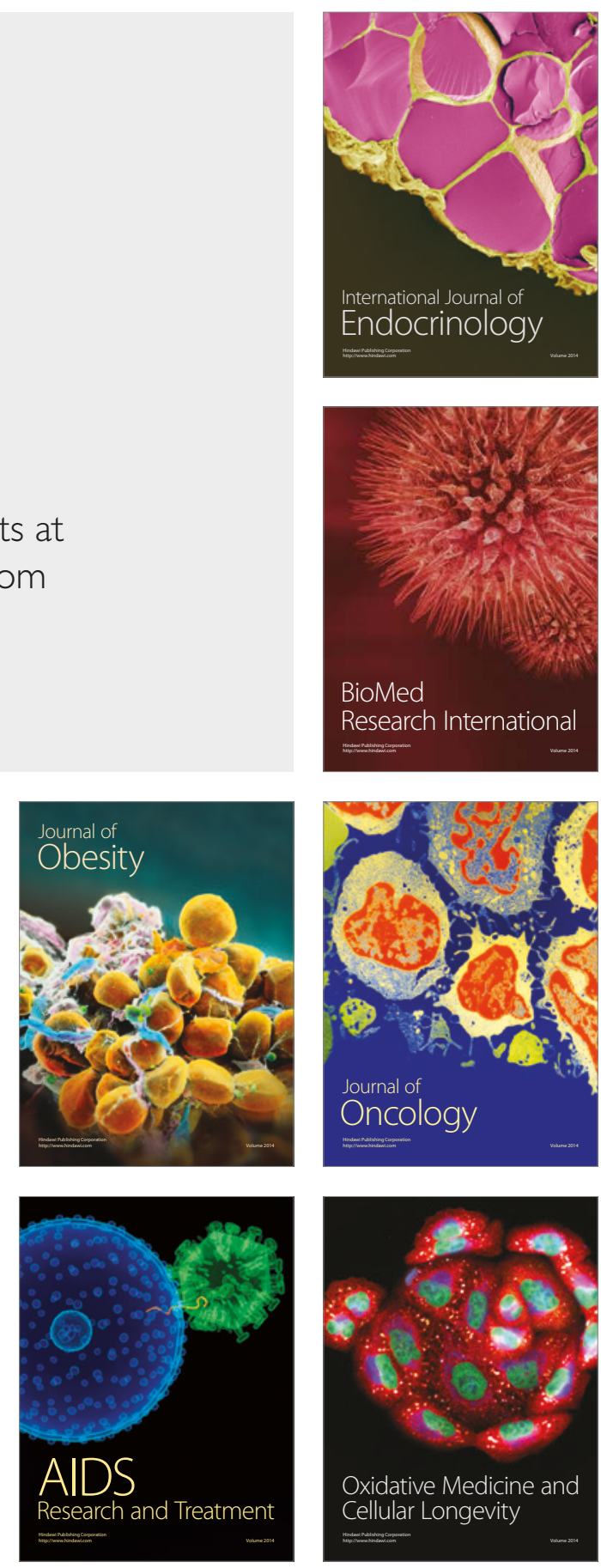